\title{
Generative processes in character classification: Evidence for a probe encoding set
}

\author{
JOHN G. SEAMON and CHARLES E. WRIGHT \\ Wesleyan University, Middletown, Connecticut 06457
}

\begin{abstract}
The role of rehearsal in a varied set memory scanning task was investigated by asking the subjects to rehearse subvocally the one to four target characters cyclically at a self-paced rate until the probe was presented. After making a manual positive or negative response to the probe, the subjects reported the last item rehearsed before the probe was presented. The results indicate that, when the last rehearsed item matched the probe, RTs were significantly faster than when it differed. Mean RTs over target set size were generally well fit by linearly increasing RT functions, with comparable slope values for negative responses and positive responses when the last rehearsal was the same as or different from the probe. The data suggest that rehearsal may reduce the duration of the probe encoding stage through some mechanism of pathway activation by providing the subject with a generated representation of what may appear next as a probe.
\end{abstract}

In a character classification task, a subject typically is presented with a sequence of alphanumeric characters to hold in immediate memory followed by a blank interval and a single probe character. The subject is required to indicate as quickly as possible if the probe is a member of the target array by making an appropriate manual response. While the data may suggest a high-speed, serial exhaustive memory comparison process (see Atkinson, Hermann, \& Wescourt, 1974; Nickerson, 1972; Shiffrin \& Schneider, 1974; Sternberg, 1969 for reviews of the empirical findings), subjects are generally unaware of how they are capable of responding so quickly and accurately trial after trial. Occasionally, however, a subject says something insightful about the nature of his processing. Although the comments may be considered no more than hints, they are worth noting. Such was the experience of the first author several months ago after completing an experimental session with a highly practiced subject. The subject was concerned over variability in his performance, having noted that he was faster on some trials than on others. This variability, he felt, was due to the probe catching him at either appropriate or inappropriate times during his rehearsal of the target characters, i.e., when the probe matched the last rehearsed item he was set and responded quickly, whereas when the probe differed from the last rehearsed item he was not set and the response seemed to be slower. Subsequent conversations with other practiced subjects have yielded similar observations.

This research was supported by a Wesleyan University faculty grant awarded to the first author. Appreciation is expressed to David Todd and George Zepko for making the facilities at the University Computer Center available and to reviewers for their thoughtful comments. The second author is now at the University of Michigan. Requests for reprints should be sent to John G. Seamon, Department of Psychology, Wesleyan University, Middletown, Connecticut 06457.
If the subjective reports are correct, rehearsal activity may affect the processes employed in the character classification task. Unless the target characters are presented rapidly with a very short temporal delay before the presentation of the probe, the target characters must be maintained in immediate memory by some form of rehearsal to insure fast and accurate response decisions. The role of this rehearsal has not been systematically investigated, although several functions may be suggested. The present experiment sought to examine possible roles for rehearsal in a varied set memory scanning paradigm, wherein subjects are presented with a sequence of one to four letters, followed by a blank interval and a single letter probe. By controlling the order of rehearsal and determining the last character rehearsed before the probe, it should be possible to determine if rehearsal serves primarily to (a) maintain representations in immediate memory, (b) strengthen representations, or (c) generate representations of possible probes if a circular distance function is computed between the probe character and the last character rehearsed such that the distance (D) is 0 when these characters are the same, 1 when the probe is the character rehearsed just previous to the last rehearsal item, and so on. With this distance function, predictions can be made about the reaction times (RTs) for positive and negative responses over target set size for different rehearsal models.

Model A: Rehearsal may serve only to maintain information in a readily available state to prevent forgetting before the probe arrives. This is apparently the view tacitly assumed by most workers in this area. If rehearsal's only function is to maintain information in immediate memory, data consistent with Sternberg's serial exhaustive scanning model may be expected. The RT should increase linearly over target set size at the same rate for positive and negative responses, and there should be no effect on either the 
slopes or intercepts of the RT functions for any value of $D$.

$$
\begin{array}{ll}
\text { A. } & \text { RT (No) }=a^{b}+b s \\
\text { RT (Yes) }=a+b s
\end{array}
$$

where $s$ is target set size and $a^{\prime}$ is never less than a.

Model B: Rehearsal may be viewed not only as a means of maintaining information, but also as a means of varying the strength of individual traces (Baddeley \& Ecob, 1973; Nickerson, 1972). Nickerson's (1972, pp. 284-285) trace strength model of character classification holds that traces in immediate memory decay rapidly, rehearsal reactivates these traces, increasing their strength, and RT is an inverse function of trace strength which is determined by the recency of the last rehearsal. The mean RT is assumed to increase with target set size because, as the number of targets increases, the time between trace reactivations lengthens, and average trace strength declines. If rehearsal can strengthen as well as maintain traces and decisions are based on a parallel self-terminating process, as Nickerson (1972) suggests, there may be no effect of target size upon RT when traces are equated on the basis of $D$, the rehearsal distance function, if it is assumed that an item is brought to full strength following each rehearsal as shown in Nickerson' Figure 5 (p. 285). The typically observed linear RT function for positive responses is produced by averaging $\mathrm{RT}$ over all values of $\mathrm{D}$ at each target size. A parallel negative RT function is obtained if negative responses are made by default (Nickerson, 1972).

$$
\text { B. } \quad \begin{array}{ll}
\text { RT (No) }=c^{\prime} \\
\text { RT (Yes) }=c_{d}
\end{array}
$$

where $c_{d}$ varies directly as a function of $D$ and $c_{d}$ is always smaller than $c^{\prime}$.

Model C: Rehearsal may serve to maintain information, but, more importantly, it may function as a generative process in which the subject is capable of generating models of what may appear next as the probe. There is ample evidence that subjects are able to generate representational models in stimulus classification tasks employing single letters (Posner, Boies, Eichelman, \& Taylor, 1969) or faces and names (Tversky, 1969), although direct evidence for generative processes in memory scanning has been lacking. This latter possibility is of interest in that it might suggest that generative processes, if present, may function like a correct guess in a fixed set memory paradigm, where trials consist of one probe after another, or like a correct expectancy in a choice RT task. Shiffrin and Schneider (1974) report faster RTs for correctly guessed probes than for incorrectly guessed probes in a fixed set paradigm, with the RT functions for both types of guesses linearly increasing over target set size at approximately the same rate. This strongly implies that expectancy affects either probe encoding or response organization, but not the memory comparison process. Hinrichs and Krainz (1970) and Hacker and Hinrichs (1974) found that RTs were faster to correct predictions than to incorrect predictions in a choice RT task with stimulus rather than response anticipation producing the major effect. Taken together, these data are not inconsistent with the results of Eichelman (1970) and Posner and Boies (1971), who report that the encoding time of a single letter was reduced if that character was preceded by an identical letter. Posner and Boies suggest that the first character may provide a model of what the subject is looking for and reduce the encoding time for the second character through some mechanism of pathway activation. A similar process may be involved in memory scanning to the extent that rehearsal of the target characters may produce models of what may appear next as the probe.

If rehearsal can serve as a generative process within the context of Sternberg's serial exhaustive scanning model, different predictions are obtained if it is assumed that the generated representation suffers continuous decay until regeneration (Model $\mathrm{C}_{1}$ ) or else decays so rapidly as to appear as a two-state process in which it is either generated and present or not generated and absent (Model $\mathrm{C}_{2}$ ).

$$
\begin{array}{ll}
\mathrm{C}_{1} & \text { RT (No) }=\mathrm{a}^{\prime}+\mathrm{bs} \\
& \text { RT (Yes) }=\mathrm{a}_{\mathrm{d}}+\mathrm{bs}
\end{array}
$$

where $a_{d}$ varies directly as a function of $D$ and $a^{\prime}$ is never smaller than the largest value of $a_{d}$. In Model $C_{2}$ RT should once more increase with target set size but be affected by $D$ only in those instances when the probe is the same as the last rehearsed item.

$$
\begin{array}{ll}
\mathrm{C}_{2} & \text { RT (No) }=\mathrm{a}^{\prime}+b s \\
\text { RT (Yes, D } \neq 0)=a+b s \\
\text { RT (Yes, D =0) }=a_{0}+b s
\end{array}
$$

where $a_{o}$ is less than a and $a^{\prime}$ is never less than $a$.

It may be argued that a processing model which posits an effect of rehearsal upon the slope value of the linear function should be presented. Bertelson (1961) and Keele (1969) have suggested that RTs to repeated stimuli in a choice RT task are faster than those to nonrepeated stimuli because subjects are able to bypass the memory comparison stage by simply remembering their preceding response (see Keele, 1973, pp. 91-93, for a review). Such an assumption might be expected to show a major effect upon the slope values for probes which match the last rehearsed item and those which do not. However, the previously cited finding of Shiffrin and Schneider (1974) that scanning was not bypassed, as well as the general report in the literature of parallel positive and 
negative functions for RT over target size (Sternberg, 1969), seem to indicate that the memory bypass assumption, if correct, is limited in its generality to other paradigms.

\section{METHOD}

\section{Subjects}

The subjects were four Wesleyan undergraduates and the two experimenters. All subjects were volunteers who had participated in a previous memory scanning task employing words as stimuli and probes. The experiment was conducted over four sessions, with the subjects paid $\$ 1.50$ per session.

\section{Apparatus}

The test stimuli employed in this study were visual characters randomly chosen from the set $A, B, D, E, F, G, H$, $\mathrm{I}, \mathrm{J}, \mathrm{K}, \mathrm{L}, \mathrm{M}, \mathrm{N}, \mathrm{Q}, \mathrm{R}, \mathrm{T}, \mathrm{Y}$, with the remaining characters excluded because of high similarity to either other characters in the set or their lowercase counterparts. The test stimuli were presented on a Digital Equipment graphics display terminal (GT-40), with each character approximately $2 \mathrm{~mm}$ in height on the display screen. The GT -40 and a standard keyboard used by the subject for responding were controlled by a PDP 11/05 computer.

All subjects participated individually. Their arms rested on a table and their index fingers were lightly placed on the positive and negative response buttons of the keyboard. The display screen was located at eye level behind the keyboard approximately $60 \mathrm{~cm}$ from the subject. Responses were made by pressing the appropriate "Yes" or "No" button with the right or left index finger, respectively. These buttons were $45 \mathrm{~cm}$ apart and separate from the other buttons on the keyboard. The subjects also responded to a question about the last rehearsed item by pressing one of five numbered buttons at the top of the keyboard.

\section{Procedure}

The experiment was conducted over a 6-day period with a session on each of the first and last 2 consecutive days. The four experimental sessions were divided into two sessions with all target and probe characters displayed in uppercase (pure condition) and two sessions with targets displayed in uppercase but probes shown in upper- or lowercase with equal frequency (mixed condition). Session type was alternated for each subject for each day, with half of the subjects starting in the pure condition and the other half starting in the mixed condition. Only data from the pure sessions will be presented in this paper. The data from the mixed sessions are entirely consistent with the results from the pure sessions, including tests of significance. They are, however, based on half the number of observations and less orderly than the pure session data. Additional observations are being made on the mixed condition, and the data will be presented at a subsequent time.

Over the two pure sessions, each subject was presented with a total of 288 trials consisting of 72 trials for each target set size of one to four characters, with half of the correct responses in each target set size positive and half negative. For positive responses, all serial positions within the target array were equally probable. In addition, target set size response, and serial position for positive probes were randomized from trial to trial.

A trial consisted of the sequential presentation of the one to four characters in the target set for $1 \mathrm{sec}$ each at a point in the top center of the display screen. The target array was preceded and succeeded by an auditory beep. A 2.5 -sec blank interval followed the second beep, during which time the subject was instructed to fixate on the probe presentation point which was located $10 \mathrm{~cm}$ below that of the target and to rehearse subvocally the target characters cyclically in the order shown at a comfortable self-paced rate. With the presentation of the probe character, the subject was required to indicate whether it was a member of the target array by making a manual yes or no response as quickly and as accurately as possible.

After making a response decision, the subject was presented with a listing in multiple-choice format of all of the target characters from the array just shown along with an additional choice labeled "DON'T KNOW." The subject's task was to select the number of the alternative that corresponded to the item in the target array which was the last item rehearsed prior to the presentation of the probe. If the subject was unsure of his last rehearsal or otherwise unable to make an appropriate character selection, he was instructed to select the DON'T KNOW alternative, with no penalties attached to this selection. The responses to the multiple-choice test were made manually via the subject's keyboard, with a visual prompt presented on the screen if a selection had not been made within $5 \mathrm{sec}$. Subjects did not report any difficulty in either subvocally rehearsing the characters or monitoring their rehearsal.

The rehearsal question was followed by a 2 -sec intertrial interval and the next trial. The subject could interrupt the sequence of trials after any trial by pressing the space bar on the keyboard during the intertrial interval. A subject was told to take this option if he felt fatigued or if he had made an error. Error trials, when present, were repeated at a randomly determined point in the trial sequence.

\section{RESULTS}

An analysis of error percentages by target set size, response, and distance function is presented along the abscissa of Figure 1. Errors occurred on $6.60 \%$ of the total trials and were excluded from the RT analysis. Similarly excluded from the data analysis were four trials $(.21 \%)$ in which a subject could not identify the last rehearsed item and selected the DON'T KNOW alternative on the rehearsal test.

Mean latencies for correct responses were obtained from the individual subject means for each target size, response condition, and rehearsal distance function and are shown in Figure 1 along with the best-fitting linear equations. It may readily be seen that (a) RT increases as a monotonic function of target set size for positive and negative responses, (b) positive responses are faster than negative responses, (c) the rehearsal distance function has an effect on RT such that when $D=0, R T$ is less than all other values of $D$ which do not influence RT, and (d) the RT functions for positive responses for $D=0$ and $D \neq 0$ and for negative responses are generally well fit by linear functions with comparable slope values which show that RT for different values of $\mathrm{D}$ did not interact with target size.

The above observations were confirmed by an analysis of variance of RT over target set size and response condition which found that $\mathrm{RT}$ increased significantly over target size, $F(3,15)=76.57, p<.001$, $\mathrm{MS}_{\mathrm{e}}=311.32$, positive responses were faster than negative responses, $F(1,5)=20.49, \mathrm{p}<.01, \mathrm{MS}_{\mathrm{e}}=$ $1,349.55$, and a marginal interaction of target size and response condition was suggested, $F(3,15)=2.52$, $.10>\mathrm{p}>.05, \mathrm{MS}_{\mathrm{e}}=191.25$. The latter trend toward 


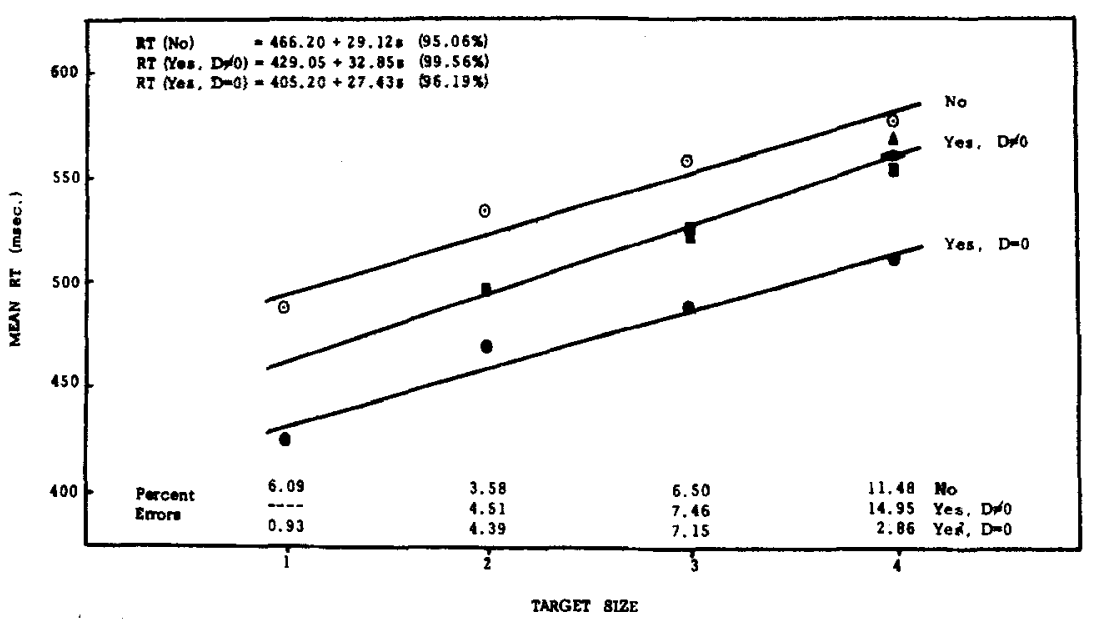

Figure 1. Mean RT as a function of target size for positive responses when $\mathrm{D}=0$ (filled circles), $\mathrm{D}=1$ (filled squares), $D=2$ (filled triangles), $D=3$ (filled diamond), and negative responses (open circles). Best-fitting linear functions were obtained by the method of least-squares and are shown with the percentage of variance acounted for by linear regression. Error percentages as a function of response, rehearsal distance, and target size are presented along the abscissa. an interaction may be observed in Figure 1 by pooling the positive responses over all levels of $\mathrm{D}$. The obtained RT function would have a steeper slope than that for either $\mathrm{D}=0$ or $\mathrm{D} \neq 0$ alone. Nonparallel response functions have been observed in several instances where a short interstimulus interval has been employed (Cavanagh, Note 1; Corballis, Kirby, \& Miller, 1972; Forrin \& Morin, 1969). These studies have shown steeper RT functions for positive responses than for negative responses and fairly strong recency effects for the last item in the target. It may be that either recent rehearsal or exposure is sufficient to produce the nonparallel response functions, while the more typical finding of parallel functions is obtained when interstimulus intervals of more than $1 \mathrm{sec}$ are used and no rehearsal instructions are provided.

An analysis of variance applied to the positive response data for RT over Target Sizes 2.4 for the individual subject means when $\mathrm{D}=0$ and $\mathrm{D} \neq 0$ again showed a significant effect of target size upon RT, $\mathrm{F}(2,10)=37.11, \mathrm{p}<.001, \mathrm{MS}_{\mathrm{e}}=233.61$, and, most importantly, a significant difference between RT when $D=0$ and $D \neq 0$, with the former faster than the latter, $F(1,5)=9.81, \mathrm{p}<.05, \mathrm{MS}_{\mathrm{e}}=1,320.49$, and no hint of an interaction between distance function and target size, $\mathrm{F}(2,10)=1.45, \mathrm{p}>.20, \mathrm{MS}_{\mathrm{e}}=298.94$. Lastly, an analysis of RT when D $=0$ over Target Sizes 1.4 again found a strong effect of number of targets upon RT, $F(3,15)=27.12, \mathrm{p}<.001, \mathrm{MS}_{\mathrm{e}}=288.78$.
A comparison of the expected and observed frequency of occurrence of positive responses for each value of $D$ at each target size is presented in Table 1 . When analyzed by $\chi^{2}$, the distributions of the differences between the probe and the last rehearsal were not significantly different from chance for Target Sizes 2 and 3 (both ps $>.10$ ). There was, however, a significant departure in Target Size $4(p<.05)$ which indicates that the subjects had a higher frequency of $D=0$ and a lower frequency of $D=3$ than would be expected from chance. The reason for the discrepancy in Target Size 4 is not apparent and it would not appear to be a strong factor, as significant differences were not observed in the other instances.

Of principal importance in Table 1 is the finding that, as target size increased, the probability that the probe matched the last rehearsal systematically decreased from $100 \%$ at Target Size 1 to $29.16 \%$ at Target Size 4, with corresponding reductions for other values of D over target size as well. These data, together with the results of the RT analysis, show that, while the probability of occurrence for any value of $\mathrm{D}$ decreases over target size, RT increases linearly over target set size at approximately the same rate for each value of $D$.

An analysis of the expected and observed frequencies of the serial position of the last rehearsed character in the target array is presented in Table 2. A $\chi^{2}$ comparison of expected and observed frequencies found significant departures from chance in Target Sizes $2(p<.001)$,

Table 1

A Comparison of Expected and Observed Frequencies for the Difference Between the Probe and Last Rehearsal

\begin{tabular}{|c|c|c|c|c|c|c|c|}
\hline \multirow{2}{*}{$\begin{array}{c}\text { Target } \\
\text { Size }\end{array}$} & \multirow{2}{*}{$\begin{array}{c}\text { Total } \\
\text { Frequency }\end{array}$} & \multirow{2}{*}{$\begin{array}{l}\text { Don't } \\
\text { Knows }\end{array}$} & \multirow[b]{2}{*}{ Frequency } & \multicolumn{4}{|c|}{ Probe and Last Rehearsal Difference } \\
\hline & & & & 0 & 1 & 2 & 3 \\
\hline 1 & 215 & 1 & $\begin{array}{l}\text { Expected } \\
\text { Observed }\end{array}$ & $\begin{array}{l}215 \\
215\end{array}$ & & & \\
\hline 2 & 215 & 1 & Expected & 107.5 & 107.5 & & \\
\hline 2 & 215 & 1 & Observed & 109 & 106 & & \\
\hline 3 & . 214 & 2 & $\begin{array}{l}\text { Expected } \\
\text { Observed }\end{array}$ & $\begin{array}{l}71.3 \\
65\end{array}$ & $\begin{array}{l}71.3 \\
77\end{array}$ & $\begin{array}{l}71.3 \\
72\end{array}$ & \\
\hline 4 & 216 & 0 & $\begin{array}{l}\text { Expected } \\
\text { Observed }\end{array}$ & $\begin{array}{l}54 \\
68\end{array}$ & $\begin{array}{l}54 \\
61\end{array}$ & $\begin{array}{l}54 \\
48\end{array}$ & $\begin{array}{l}54 \\
39\end{array}$ \\
\hline
\end{tabular}


$3(\mathrm{p}<.01)$, and $4(\mathrm{p}<.05)$. These differences are not unexpected if it is assumed that the subjects were rehearsing the target characters over the constant blank interval at a fairly uniform rate, with the probe, because of these uniformities, apparently more likely to catch the subjects at the beginning of a new rehearsal cycle than at the end of an old. Use of a variable, rather than a constant blank, interval should alter the frequency distribution of last rehearsals at each serial position.

The finding that the last rehearsal was not evenly distributed over all serial positions within the target array may have a bearing on the RT data. Since RT is faster when $D=0$ than when $D \neq 0$ and there were significant serial position effects such that the probability of being the last rehearsal item decreased from Serial Position 1 to $\mathrm{N}$ over all target sizes, it may be expected that an analysis of positive RTs plotted as a function of serial position and target size would show differences as a function of the serial position of the probe in the target. Such is indeed the case, as shown in the left-hand portion of Table 3. Significant serial position effects (all ps $<.01$ ) were observed for Target Set Sizes 24 by analyses of variance. One might expect that the position effects would be reduced if RT was analyzed as a function of target size, serial position, and distance function between the probe and last rehearsal. This was done for $D=0$ and $D=1-3$ and is also presented in Table 3. The data for $D=0$ and $\mathrm{D}=1-.3$ were transformed by a $\log$ transformation to eliminate heterogeneity of variance with subsequent analyses of variance comparing mean RTs for serial position and level of $D$ at each target size. Contrary to expectation, interaction effects were not observed in any instance (all ps $>.20$ ). These data, however, are not conclusive, as all subjects did not contribute equally to the $D=0$ means. Some subjects did not yield any observations for certain serial positions under $\mathrm{D}=0$. It would appear that, whatever the origin of the RT serial position effects, the effect of rehearsal distance function does not interact with target set size or serial position within a target. Serial position effects, like those observed in this experiment, have been found by Klatzky, Juola, and Atkinson (1971), Klatzky and Smith (1972), and Seamon (Note 2). In the latter case, Sternberg's (Note 3) test for a self-terminating search was applied to the data, with no evidence found to reject a serial exhaustive comparison process.
Table 2

A Comparison of Expected and Observed Frequencies for Serial Position of Last Rehearsal in Target Array

\begin{tabular}{|c|c|c|c|c|c|c|}
\hline \multirow{2}{*}{$\begin{array}{l}\text { Target } \\
\text { Size }\end{array}$} & \multirow{2}{*}{$\begin{array}{l}\text { Total } \\
\text { Freq }\end{array}$} & \multirow[b]{2}{*}{ Frequency } & \multicolumn{4}{|c|}{ Serial Position in Target } \\
\hline & & & 1 & 2 & & 3 \\
\hline 1 & 215 & Expected & 215 & & & \\
\hline & & Fynec & $\begin{array}{l}215 \\
1075\end{array}$ & 1075 & & \\
\hline 2 & 215 & $\begin{array}{l}\text { Expected } \\
\text { Observed }\end{array}$ & $\begin{array}{l}107.5 \\
138\end{array}$ & 77 & & \\
\hline 3 & 214 & $\begin{array}{l}\text { Expected } \\
\text { Observed }\end{array}$ & $\begin{array}{l}71.3 \\
89\end{array}$ & $\begin{array}{l}71.3 \\
77\end{array}$ & $\begin{array}{l}71.3 \\
48\end{array}$ & \\
\hline 4 & 216 & $\begin{array}{l}\text { Expected } \\
\text { Observed }\end{array}$ & $\begin{array}{l}54 \\
63\end{array}$ & $\begin{array}{l}54 \\
56\end{array}$ & $\begin{array}{l}54 \\
61\end{array}$ & $\begin{array}{l}54 \\
36\end{array}$ \\
\hline
\end{tabular}

\section{DISCUSSION}

It has been shown that RT is significantly affected by the target set size, response condition, and rehearsal distance function in a memory scanning task. When latencies for positive responses were examined as a function of $D$, it was found that RT increased linearly over target size at a comparable rate for all values of $D$. When the probe was the same as the last rehearsed item, RTs were significantly faster than when different, and, if different, it did not matter whether the distance between the probe and the last rehearsed item was one, two, or three character spaces in the rehearsal sequence.

In light of the present data, the various rehearsal models presented earlier may now be critically reviewed. Model A assumed that rehearsal's only function was to maintain information in immediate memory until the probe arrived. Contrary to Model A, the results show that rehearsal processes clearly have an effect upon RT and thus do more than simply prevent forgetting of the target characters. Likewise without support is the strength model put forth by Nickerson (1972), which posits that rehearsal affects trace strengths. Model B, which predicts no effect of target size on RT when the traces are equated on the basis of recency of last rehearsal, is not supported by the finding of significantly increasing functions of RT over target size for $D=0$ and $D \neq 0$ and by the fact that, apart from the difference between $D=0$ and $D \neq 0$, RT did not vary monotonically with levels of $\mathrm{D}$, which is at odds with the recency assumption.

It might appear possible to construct a strength model which yields monotonically increasing RT functions for each level of $D$ over target size if, accord-

Table 3

Mean RTs by Serial Position of Probe in Target Array

\begin{tabular}{|c|c|c|c|c|c|c|c|c|c|c|c|c|}
\hline \multirow{2}{*}{$\begin{array}{l}\text { Target } \\
\text { Size }\end{array}$} & \multicolumn{4}{|c|}{ Yes Trials for $D=0-3$} & \multicolumn{4}{|c|}{ Yes Trials for $D=0$} & \multicolumn{4}{|c|}{ Yes Trials for $D=1-3$} \\
\hline & 1 & 2 & 3 & 4 & 1 & 2 & 3 & 4 & 1 & 2 & 3 & 4 \\
\hline 1 & 426 & & & & 426 & & & & & & & \\
\hline 2 & 457 & 506 & & & 463 & 497 & & & 454 & 516 & & \\
\hline 3 & 477 & 527 & 533 & & $457^{*}$ & 504 & $517^{\prime}$ & & 490 & 542 & 540 & \\
\hline 4 & 488 & 543 & 562 & 585 & 462 & 553 & 496 & $604^{* *}$ & 492 & 541 & 586 & 587 \\
\hline
\end{tabular}

Note. All cells include the subject means for six subjects except for the cells with single or double asterisks which include subject means for only five and four subjects, respectively. 
ing to Nickerson (1972, pp. 284-285), strength is a function of both recency and frequency such that as target set size increases, rehearsal frequency decreases and strength may vary inversely with target set size even when recency has been held constant. However, RT should still vary monotonically over levels of $D$, which it clearly does not do in Figure 1. To account for these data, it would appear necessary to disregard recency of rehearsal per se and make the strong assumptions that the level of strength achieved by the last rehearsal is a function of the number of other items being rehearsed and that whatever the level of strength achieved by the last rehearsal, trace strength decays to the same level as all other currently nonrehearsed items in the target set as soon as the next rehearsal is made. As such, the number of items in the target set would influence the level of trace strength for a given rehearsal, but not its subsequent rate of decay. These changes substantially alter Nickerson's originally simple trace strength alternative to Sternberg's serial scanning model.

The last class of models are those which argue that rehearsal could serve as a generative process to facilitate encoding of the probe by providing models of what the probe may be. While there is no support for Model $\mathrm{C}_{1}$, which assumes, like the strength model, that RT will vary directly with level of $D$, the data are not inconsistent with Model $\mathrm{C}_{2}$, which holds that RT will increase over target size and be affected by $\mathrm{D}$ only in those instances when the probe is the same as the last rehearsal item. Equations 7, 8, and 9, which describe Model $\mathrm{C}_{2}$ are graphically portrayed in the RT functions in Figure 1.

Given that rehearsal processes have an effect on $\mathrm{RT}$, it is important to ask what processing stage is affected by the rehearsal of the target characters. If we assume Sternberg's processing stages approachwhich posits independent and nonoverlapping stages of probe encoding, memory comparison, and response organization-in this task, it is clear that we may reject the memory comparison stage as the locus of the effect. The rate of scanning in this stage may be inferred from the slopes of the RT functions for positive and negative responses. Since the RT functions in Figure 1 are well described by a set of linear functions with approximately the same slopes, it would appear that this stage was the same for positive responses at all values of $D$ and for negative responses. These data would suggest a high-speed serial exhaustive comparison process.

Also capable of being rejected is the possibility that rehearsal enabled subjects to make their responses faster through some form of motor set. While this may be a plausible assumption in other circumstances, such as the choice RT or fixed target paradigms, we do not think it is appropriate in this situation, where the response probabilities are equal with trial-to-trial randomization and, most importantly, where the subject is changing his "set" from moment to moment by cycling the target array through a rehearsal buffer one item at a time. A motor set for the yes response would find the subjects making faster responses to positive probes in all conditions with no effect of level of $D$. While there is a small, but reliable, difference between positive RTs when $D \neq 0$ and negative $R T$ s which may be attributable to this response set, the difference in $\mathrm{RT}$ between $D=0$ and $D \neq 0$ cannot be. This latter difference would appear to be a function of the probe encoding stage.

It is argued that rehearsal affects the duration of the probe encoding stage by providing the subject with models of what may appear next. It is possible that generated models affect subsequent processing in a number of different ways. It may be that processing time of a probe is reduced when the subject has generated the appropriate representation beforehand. This would demonstrate that generation has a facilitative effect upon later processing and may be described in terms of a pathway activation model (Posner \& Boies, 1971), whereby generating representations may be viewed as the preparatory activation of visual analyzers such as those proposed by Selfridge and Neisser (1960) (Nickerson, 1972). We believe, as others have argued previously, that generated information must, to some extent, be similar in terms of structures and processes to information provided by an external source. Alternatively, it may be that generating the appropriate model does not speed up later processing, but rather generating an inappropriate model slows it down. This hypothesis would hold that generation could have an interfering effect upon processing such that the subject has begun to preprocess the wrong stimulus. Lastly, generative processes could have both a facilitation and interference effect upon subsequent processing depending upon the relationship between the generated representation and the probe stimulus. Facilitation occurs when they are the same and interference occurs when they are different. The present data cannot differentiate these hypotheses, although some support for the facilitation hypothesis is found in an electrophysiological study of letter matching by Posner, Klein, Summers, and Buggie (1973). They suggest that the encoding time of a second letter is reduced when it matches the first because it reaches a "limited capacity (conscious) mechanism" (Posner et al., 1973) earlier than if the second letter was a mismatch. Interestingly, there is some evidence in the literature to suggest that RT may be reduced by the presentation of information on the preceding trial which is not identical to that called for on the current trial. This was observed by faster recognition RTs for words preceded by semantically related words than unrelated words (Meyer, Schvaneveldt, \& Ruddy, 1974) and by faster recall RTs when the same semantic categories, but different semantic instances, were probed 
on successive trials (Seamon, 1973). If information is represented in a highly interconnected neural network, the concept of pathway activation may be relevant in these instances as well.

Lastly, Shiffrin and Schneider (1974) raise the question of whether a one-element expectancy buffer is appropriate. Our data are certainly consistent with this notion and preliminary work in our lab has shown similar results when four-letter words are used as stimuli and probes. However, it is not clear if this is a structural limitation of the processing system, or the result of our instructions to rehearse the target array one item at a time. Hacker and Hinrichs (1974) have shown that RTs are faster to correct first and second predictions than incorrect predictions in a choice RT task, which would appear to suggest that a one-element buffer may not always be correct. Additional research may determine the limit on the amount of information that may be generated at a given period of time. We suspect, however, that a better understanding of the role of the generated representations will be necessary before answering many questions about what a subject is doing while waiting for an event.

\section{REFERENCE NOTES}

1. Cavanagh, P. Differential effects of rehearsal strategy on negative and positive slopes in item recognition. Paper presented at the annual meeting, Psychonomic Society, Boston, November 1974.

2. Seamon, J. G. Memory scanning through word and nonword letter strings. Paper presented at the annual convention, American Psychological Association, New Orleans, August 1974.

3. Sternberg, S. Evidence against self-terminating memory search from properties of RT distributions. Paper presented at the annual meeting, Psychonomic Society, St. Louis, November 1973.

\section{REFERENCES}

Atikinson, R. C., Herrmann, D. J., \& Wescourt, K. T. Search processes in recognition memory. In $R$. L. Solso (Ed.), Theories in cognitive psychology. Washington, D. C: Winston, 1974.

BADDeley, A. D., \& EcoB, J. R. Reaction time and shortterm memory: Implications of repetition effects for the highspeed exhaustive scan hypothesis. Quarterty Journal of Experimental Psychology, 1973, 25, 229-240.

Bertelson, P. Sequential redundancy and speed in a serial two-choice responding task. Quarterly Journal of Experimental Psychology, 1961, 12, 90-102.

Copbatlis, M. C., KIrby, J., \& Miller, A. Access to elements of a memorized list. Journal of Experimental Psychology, 1972. 94, 185-190.

Eichelman, W. H. Stimulus and response repetition effects for naming letters at two response-stimulus intervals. Perception \& Psychophysics, 1970, 7, 94-96.

ForRIN, B., \& Morin, R. E. Recognition times for items in short- and long-term memory. In W. G. Koster (Ed.), Attention and performance II. Amsterdam: North Holland, 1969. Pp. 126-141.

Hacker, M. J., \& Hinrichs, J. V. Multiple predictions in choice reaction time: A serial memory scanning interpretation. Journal of Experimental Psychology, 1974, 103, 999-1005.

Hinrichs, J. V., \& Krainz, P. L. Expectancy in choice reaction time: Anticipation of stimulus or response? Journal of Experimental Psychology, 1970, 85, 330-334.

KeEle, S. W. Repetition effect: A memory-dependent process. Journal of Experimental Psychology, 1969, 80, 243-248.

Keele, S. W. Attention and human performance. Pacific Palisades, Calif: Goodyear, 1973.

Klatzky, R. L., Juola, J. F., \& Atrinson, R. C. Test stimulus representation and experimental context effects in memory scanning. Joumal of Experimental Psychology, 1971, 87, 281-288.

KLAtzky, R. L., \& SMith, E. E. Stimulus expectancy and retrieval from short-term memory. Journal of Experimental Psychology, 1972, 94, 101-107.

Meyer, D. E., Schvaneveldt, R. W., \& Ruddy, M. G. Loci of contextual effects in visual word recognition. In P. Rabbitt (Ed.), Attention and performance V. New York: Academic Press, 1974.

Nickerson, R. S. Binary-classification reaction time: A review of some studies of human information-processing capabilities. Psychonomic Monograph Supplements, 1972, 4, no. 17(Whole No. 65).

Posner, M. I., \& Bores, S. J. Components of attention. Psychological Review, 1971, 78, 391-408.

Posner, M. I., Boies, S. J., Eichelman, W. H., \& TaYlor, R. L. Retention of visual and name codes of single letters. Journal of Experimental Psychology Monograph, 1969, 79, No. 1, Part 2.

Posner, M. I., Klein, R., Sumgers, J., \& Buggie, S. On the selection of signals. Memory \& Cognition, 1973, 1, 2-12.

SeAmon, J. G. Retrieval processes for organized long-term storage. Journal of Experimental Psychology, 1973, 97, 170-176.

Selfridge, O. G., \& Neisser, U. Pattern recognition by machine. Scientific American, 1960, 203, 60-68.

Shiffrin, R. M., \& Schneider, W. An expectancy model for memory search. Memory \& Cognition, 1974, 2, 616-628.

STERngerg, S. Memory-scanning: Mental processes revealed by reaction-time experiments. American Scientist, 1969 , 57, 421-457.

TVERSxy, B. Pictorial and verbal encoding in a short-term memory task. Perception \& Psychophysics, 1969, 6, 225-233.

(Received for publication February 13, 1975; revision received May 1, 1975.) 